Ophthalmia neonatorum

\title{
Povidone-iodine prophylaxis of ophthalmia neonatorum
}

\section{J W Simon}

It is important to recognise that defining ophthalmia neonatorum in a large group of Atrican newborns is problematic

O phthalmologists practising in the developed world at the beginning of the 2 lst century may be surprised to learn that visual loss from ophthalmia neonatorum was once a major public health problem. In England in the mid-1800s, there was a hospital devoted solely to the lavage of infants with gonococcal ophthalmia. Fortunately, the work of Crede, who developed prophylaxis with $2 \%$ silver nitrate in 1881, nearly eliminated visual loss from this disease in the West. ${ }^{1}$ Unfortunately, conjunctivitis in the first month of life remains a common threat to vision in much of the developing world. In areas without prophylaxis, the incidence of ophthalmia is as high as 23\%. In Africa, between 1000 and 4000 are blinded each year. ${ }^{2}$

A variety of topical agents have been used for prophylaxis in the neonatal period. In the West, erythromycin and tetracycline have often been favoured because of their effectiveness against chlamydial infection. In the developing world, the expense of these agents, and of silver nitrate, can preclude their availability in many areas. Isenberg et al have previously demonstrated that $2.5 \%$ povidone-iodine, which can be reconsti- tuted locally from powder at minimal cost, is an appropriate alternative. Ophthalmologists may be more familiar with this agent from its use in surgical scrubs and preps. It is well tolerated and has a broader spectrum than any other agent, and there has been no resistance demonstrated to date. Because it temporarily stains the conjunctiva brown, effective administration is easy to confirm.

More important, povidone is even more effective than the more expensive alternatives. In an earlier study, Isenberg et al found the incidence of ophthalmia was reduced by $44 \%$ by povidone-iodine, compared to $34 \%$ by erythromycin and $24 \%$ by silver nitrate. ${ }^{2}$ It was more effective against chlamydia, similarly effective against gonococcus, and even effective against herpes virus and human immunodeficiency virus.

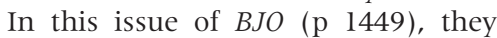
study the effectiveness of a second instillation of povidone-iodine during the first postnatal day. They conclude that the additional treatment, which increased eyelid oedema at the time of discharge, was of no benefit. Although this is essentially a negative outcome, its implication is that a single drop, obviously simpler to administer, is maximally effective.

It is important to recognise that defining ophthalmia neonatorum in a large group of African newborns is problematic. More than $18 \%$ were returned by their parents for evaluation of ocular inflammation, yet only $8-10 \%$ were culture positive. Many may have had chemical conjunctivitis, and some with staphylococcal infection may have had infections acquired postnatally at home. Some may have had viral infections, or clinically insignificant inflammation. Perhaps the most important finding is that, among more than 700 infants studied, there was not a single case of gonococcus following either one or two drop prophylaxis with povidoneiodine. Improved prenatal care may be partially responsible for decreasing the incidence of this potentially blinding infection.

The authors are to be congratulated for their contribution to the fight against childhood blindness. We encourage them to continue their research in this field.

\section{Br J Ophthalmol 2003;87:1437}

\section{Author's affiliation}

J W Simon, Lions Eye Institute, Albany Medical College, Albany, NY 12210, USA; simonj@mail.amc.edu

\section{REFERENCES}

1 Crede CSF. Die Verhutung der Augenentzundung der Neugeborenen. Arch Gynakk 1881;17:55.

2 Isenberg SJ, Apt L, Wood M. A controlled trial of povidone-iodine as prophylaxis against ophthalmia neonatorum. N Engl J Med 1995;332:562-6.

\section{Ocular surface reconstruction, amniotic membrane, and cultivated epithelial cells from the limbus}

N Koizumi, S Kinoshita

\section{Amniotic membrane has an inhibitory effect against transformation of keratocytes to myofibroblasts}

$\mathrm{S}$ evere ocular surface diseases such as Stevens-Johnson syndrome and ocular cicatricial pemphigoid, as well as a wide range of debilitating

chemical injuries, have at their core a deficiency of corneal epithelial cells at the limbus. This is because the corneal epithelial stem cells reside in this region of the eye, indicated by the presence of palisades of Vogt, ${ }^{1}$ and when the limbal epithelium is destroyed the corneal surface becomes enveloped by conjunctival tissue with superficial scarring and vascularisation often accompanying a persistent epithelial defect. To surgically reconstruct these eyes a keratolimbal graft-either from the other, healthy eye of the same patient (autograft) or from donor material (allograft) — can be carried out, ${ }^{2}$ and this approach has been beneficial in a number of situations. Autografts, however, are not an option for bilateral injuries, and even when they are feasible have drawbacks because they require a fairly large limbal graft be taken from the healthy eye which is not without risk. Allografts are not ideal as they invariably carry the risk of rejection. 
Recently, a new approach that involves the transplantation of cultivated corneal limbal cells to treat stem cell deficient eyes has provided promising results. ${ }^{3-7}$ Initial work established the premise that cells from healthy limbal tissue could be cultivated in the laboratory and grafted onto severely damaged eyes to successfully reconstruct the ocular surface. Clinically, the first application of this new surgical technology was reported by Pellegrini and colleagues in 19973; later, the concept was applied to both autologous and donor limbal epithelial cells to make epithelial cell sheets using amniotic membrane as a growth substrate and carrier. $^{4-8}$ The membrane can serve not only as an epithelial carrier but also as a healthy substrate to cover a damaged corneal stroma. Clinical data indicated that this new procedure was a good option for the repair of the corneal surface when limbal stem cells were lacking. ${ }^{4-8}$ Up to now, however, the effect on the stroma of this surgical intervention has not been discussed in any detail.

\section{The use of limbal epithelial cells expanded ex vivo on amniotic membrane holds much promise for the surgical reconstruction of severely damaged, stem cell defi- cient eyes}

This issue of BJO contains a paper by Espana and colleagues (p 1509) that details changes in the corneal stroma following ocular surface reconstruction by autologous cultivated limbal epithelial cell transplantation. In it they describe how the ocular surface was reconstructed in 16 rabbits with total limbal stem cell deficiencies. In their surgeries transplantation was with autologous cultivated (that is, ex vivo expanded) limbal epithelium on intact amniotic membrane-that is, amniotic membrane with amniotic epithelial cells left intact. The authors then investigated how corneal stromal changes related to the long term clinical outcome. Almost a year after surgery it was apparent that the restoration of a clear and transparent cornea was associated with the return of a normal corneal epithelium and complete wound remodelling in the stroma. It was also found that epithelial phenotype was linked to the fate of the amniotic membrane and changes of stromal structure. Specifically, the healed stroma under a normal corneal epithelium had the usual keratocyte cell density and did not contain inflammatory cells or any sign of vascularisation. On the other hand, stroma under an improperly healed corneal surface containing conjunctival epithelium displayed increased cellular infiltration into the stroma as well as vascularisation. The association between healed epithelium and healed stroma was especially clear on surgeries that were partially successful and which contained both clear epithelial portions and opaque conjunctivalised portions. Proper healing was also found to be related to the continued presence of the amniotic membrane. Immunostaining for collagen IV and $\alpha$-smooth muscle actin were negative when amniotic membrane was present but positive when no amniotic membrane was identified. This is in line with previous observations that the amniotic membrane itself has an inhibitory effect against transformation of keratocytes to myofibroblasts. ${ }^{9}$ Failed eyes contained no amniotic membrane, and showed diffuse vascularisation and stromal haze accompanied by a loss of lamellar structure.

Our department has experience with the type of surgeries outlined by Espana and associates, with the distinction that we use allolimbal epithelial cells cultivated on denuded (that is, without amniotic epithelium) amniotic membrane. In 1999, we started allocultivated limbal epithelial cell transplantation for total limbal stem cell deficiencies and found it highly effective for the reconstruction of total limbal stem cell deficiencies in both acute and chronic phases. ${ }^{6-8}$ Our approach involved covering the ocular surface with an already grown corneal epithelial cell sheet derived from limbal epithelial cells. Often, this turned out to be very successful in restoring good vision in patients with Stevens-Johnson syndrome and in individuals who had experienced severe chemical burns. Invariably, surgery was done during the acute phase. In the postoperative period some eyes developed epithelial opacities as a result of rejection, and in these cases we replaced the epithelium with allo-cultivated limbal epithelial cells once more. When we removed these failed graft constructs, we were somewhat surprised to find that amniotic membrane still covered most of the cornea, even a year after conjunctival invasion. The membrane, however, was easily removed at the time of the second surgery, and beneath the exposed stroma was transparent. ${ }^{10}$ On electron microscopic examination of failed grafts, we found that most of the amniotic membrane was covered by conjunctival epithelium characterised by the presence of goblet cells. In all, three failed cases were examined, and these indicated that the amniotic membrane remained intact but was partially vascularised and invaded by stromal cells.
When we examine patients on the slit lamp after allo-cultivated limbal epithelial cell transplantation we sometimes see small areas where the amniotic membrane seems to have melted or been absorbed. Though we don't know exactly what is happening, we think that this phenomenon is related to local inflammation because of allograft rejection. In our experience, the corneal stroma of the failed eyes shows little scarring and maintains its clarity fairly well. This does not correspond to the findings in the failed eyes in Espana's paper, possibly because the immunosuppressive and anti-inflammatory therapy that we administer to patients might aid retention of the amniotic membrane.

The use of limbal epithelial cells expanded ex vivo on amniotic membrane holds much promise for the surgical reconstruction of severely damaged, stem cell deficient eyes. In part this might be because cultivation on amniotic membrane preserves in the cultivated cells some characteristic signs of limbal epithelial progenitor cells. ${ }^{11}$ However, as Espana et al have shown, the widespread healing of the anterior cornea, not just the epithelium itself, is required for the successful repair of the front surface of the eye.

Br J Ophthalmol 2003;87:1437-1439

\section{Authors' affiliations}

N Koizumi, S Kinoshita, Department of Ophthalmology, Kyoto Prefectural University of Medicine, Kyoto, Japan

Correspondence to: Noriko Koizumi, MD, PhD, Department of Ophthalmology, Kyoto Prefectural University of Medicine Kawaramachi-hirokoii, Kamigyo-ku, Kyoto 602-0841, Japan; nkoizumi@ophth. kpu-m.ac.jp

\section{REFERENCES}

1 Kinoshita S, Adachi W, Sotozono C, et al. Characteristics of the human ocular surface epithelium. Prog Retin Eye Res 2001;20:639-73.

2 Holland EJ, Schwarts GS. The evolution of epithelial transplantation for severe ocular surface disease and a proposed classification system. Cornea 1996; 15:549-56.

3 Pellegrini G, Traverso CE, Franzi AT, et al. Longterm restoration of damaged corneal surfaces with autologous cultivated corneal epithelium. lancet 1997;349:990-3

4 Tsai RJ, Li LM, Chen JK. Reconstruction of damaged corneas by transplantation of autologous limbal epithelial cells. N Engl J Med 2000;343:86-93.

5 Schwab IR, Reyes M, Isseroff RR. Successful transplantation of bioengineered tissue replacement in patients with ocular surface disease. Cornea 2000;19:421-6.

6 Koizumi N, Inatomi T, Suzuki T, et al. Cultivated corneal epithelial transplantation for ocular surface reconstruction in acute phase of StevensJohnson syndrome. Arch Ophthalmol 2001;119:298-300. 
7 Koizumi N, Inatomi T, Suzuki T, et al. Cultivated corneal epithelial stem cell transplantation in ocular surface disorders. Ophthalmology $2001 \cdot 108: 1569-74$

8 Kinoshita S. Ocular surface reconstruction by tissue engineering. J Jap Ophthalmol Soc 2002; 106:837-69.
9 Choi TH, Tseng SCG. In vivo and in vitro demonstration of epithelial cell-induced myofibroblast differentiation of keratocytes and an inhibitory effect by amniotic membrane. Cornea 2001;20:197-204.

10 Nakamura T, Koizumi N, Tsuzuki M, et al. Successful regrafting of cultivated corneal epithelium using $\mathrm{AM}$ as a carrier in severe ocular surface disease. Cornea 2003;22:70-1.

11 Hernandez-Galindo EE, Theiss C, Steuhl K-P et al. Expression of $\triangle \mathrm{Np} 63$ in response to phorbol ester in human limbal epithelial cells expanded in intact human amniotic membrane. Inves Ophthalmol Vis Sci 2003:44:2959-65.

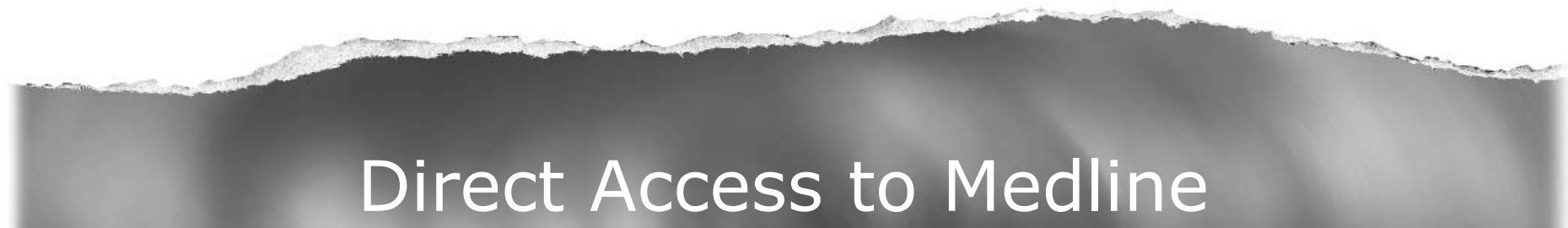

\section{Medline}

Link to Medline from the homepage and get straight into the National Library of Medicine's premier bibliographic database. Medline allows you to search across 9 million records of bibliographic citations and author abstracts from approximately 3,900 current biomedical journals.

\section{www.bjophthalmol.com}

